The Sustainable City XIII 357

\title{
SUSTAINABILITY OF HISTORICAL AND ARTISTIC HERITAGE BUILDINGS
}

\author{
ALEJANDRO ACOSTA COLLAZO \\ Architecture Department, Autonomous University of Aguascalientes, Mexico
}

\begin{abstract}
The increasing size of Aguascalientes City in Mexico has augmented its traffic flows and also the amount of parking lots during the last two decades. The challenge of this paper is to analyse the social deterioration factors of historical heritage buildings and artistic heritage buildings. Hence, in order to evaluate such facts, a multivariate statistical method was applied; thus, a cluster exploration with specific variables - including a drawing indicating a "limited part of the city" with historic (and artistic) buildings, data of building sizes, parking lots, loss of architecture, areas of orchards, vacant lots, city parks, houses, industry, services and commercial shops. The results show similar sizes of commercial shops in several street blocks. Also, it demonstrates space adjustments and alterations in historic buildings; indeed, the commercial shops have modified historic buildings and in multiple cases they have torn them down. The conclusions mention that loss of historic architecture was more common in the oldest street blocks of the city; consequently, in the historic site there's a lack of architecture built during the 16th and 17th centuries. Therefore, the most significant change is the increasing commercial shops in the "limited part of the city", substituting residential land use. As a consequence, more parking lots were built to support the increasing mobility in the city. Since cultural sustainability is at risk in Aguascalientes City, the study of changes in architectural heritage sites becomes an important part of the urban research agenda of current city planners. For these reasons, the paper can contribute to the development of social and cultural balances. Moreover, the final task is to help improve the capacity of city planning and the sustainability of historical heritage buildings and artistic heritage buildings.

Keywords: urban mobility, cultural heritage site, sustainability, loss of unique architecture.
\end{abstract}

\section{INTRODUCTION}

During the last two decades, historic buildings have suffered alterations and demolitions as a result of several factors, like a persistent urban mobility, the lack of preservation regulations and the commercial shops in historic sites (among others). In Mexico, preservation of historic heritage and artistic architecture doesn't seem to be an important matter to most of the people and city administrators, as a consequence, it becomes necessary to build new edifications to enable the needs of the population. Besides, in the city centres, there are a few regulations to avoid demolition of historic buildings. In this paper the situation regarding historical heritage buildings and artistic heritage buildings will be explained. Also, the paper includes a classification and a register of each building in a study area named "a limited part of the city". This area was established to analyse data obtained from each city block and from each building.

Aguascalientes city has lost a lot of unique architecture built during the 20th century - in absence of law protections. Most of them are now catalogued as artistic buildings to stop severe renovations, however there's still a lot do in order to prevent an effective loss of heritage buildings. Recent researches have proved that materials used originally in historic buildings are more durable than nowadays construction materials. Also, schools of architecture are now trying to convince new generations of students about the importance of historic buildings restoration and preservation, because they remind us of where we came from and who we are now. 
The paper will start with a brief history to understand the types of buildings included in the "limited part of the city". Afterwards, a multivariate statistical method will be used and at the end a cluster analysis will help us to understand similarities of city blocks and will show some steps ahead to preserve architectural heritage sites.

\section{THE FIRST SPANISH MAINLAND SETTLEMENTS AND VICEROYALTIES}

After the conquest of Mexico Tenochtitlan in 1521, Spaniards decided to search for gold and silver mainland by conquering small villages in the northern side of the Viceroyalty of New Spain. Also, an epic war occurred named: Chichimeca War and it lasted more than a century. Powell [1] mentions the real pacification of the new country occurred from 1550 to 1600 . So, it was possible to set new borderlines - according with Chichimeca tribes pacification through the 16th century. Then several religious missions were established in the northern provinces of the Kingdom of New Spain. Also, several forts and arsenals were set by the side of the main Royal Road - or Silver Road - which communicated Zacatecas with Mexico City and Veracruz, forming a main silver belt [2].

The main cities of Mexico were established during the 16th century, and also a lot of them got their names from Spain. So we can find León, Valladolid, Salamanca Guadalajara, Mérida, Monterrey, Durango, Córdoba, and some others in Mexico as well as in Spain. But in most cases, such sister cities don't have to do anything with each other, but the name. Besides, architecture built during the 16th century in Mexico is unique, but there have been several demolitions throughout the history of Mexico. The city of Aguascalientes became important for the whole country because of its geographic situation and strategic factors for the settlement. Aguascalientes village allowed security for the Silver Road and became a place where some contributions for development and prosperity of the nation took place.

Since the first settlement was also founded in the 16th century, the first main buildings were some houses situated next to the main square, a small church, an arsenal and government buildings. The central square of Aguascalientes City is Patria Square (also named Main Square), and it has been renovated since the 16th century. But during the 20th century and the beginning of the 21 st century more frequent urban renovation occurred [3]. Originally a checkerboard street system was used, just as King Philip II of Spain dictated in the zoning ordinances for new towns. It was at this place where the first buildings were built, but in the contrary: nowadays we can hardly find only one small tower of the old arsenal from the 16th century standing still. On the other hand we can still observe the checkerboard street system in the borders of each city block in the core of the "limited part of the city". A similar situation of loss of architecture happened during the 17th century. So, in the historic site there's a lack of architecture built during the 16th and 17th centuries.

\section{THE ENDING OF VICEROYALTIES AND THE 19TH CENTURY}

After the Chichimeca tribes pacification, the town administrators led up, and also promoted, the construction of new buildings. Fortunately, for the historic preservation in Aguascalientes, nowadays we can identify good Baroque architecture in the city centre built during the 18th century and in the beginning of the 19th century. In fact, the most important old churches in the city are baroque. A masterpiece of baroque architecture in the city is the main cathedral (situated in the city block 24 in Fig. 1), but also in the old quarters some Baroque churches became iconic in the core of catholic neighbourhoods. After the Mexican War of Independence in 1821, neoclassical buildings replaced the old buildings of the 16th and the 17th century in Aguascalientes City. The new academies, like San Carlos Academy, helped improve architecture with artistic principles. It's important to mention that some of 
the architects whom graduated from San Carlos decided to work in several important cities of Mexico, and not only in Mexico City. During the 19th century there were a few wars, also social movements, and strong political changes occurred in the country. So, it was very common to make tunnels to communicate different places inside a town. For example: between an altar of a church and a house, or a church with another church. People used these tunnels to escape from attacks too. Also there was a frequent destruction of religious buildings in the 19th century [4]. It also happened that some neoclassic altars replaced baroque altars.

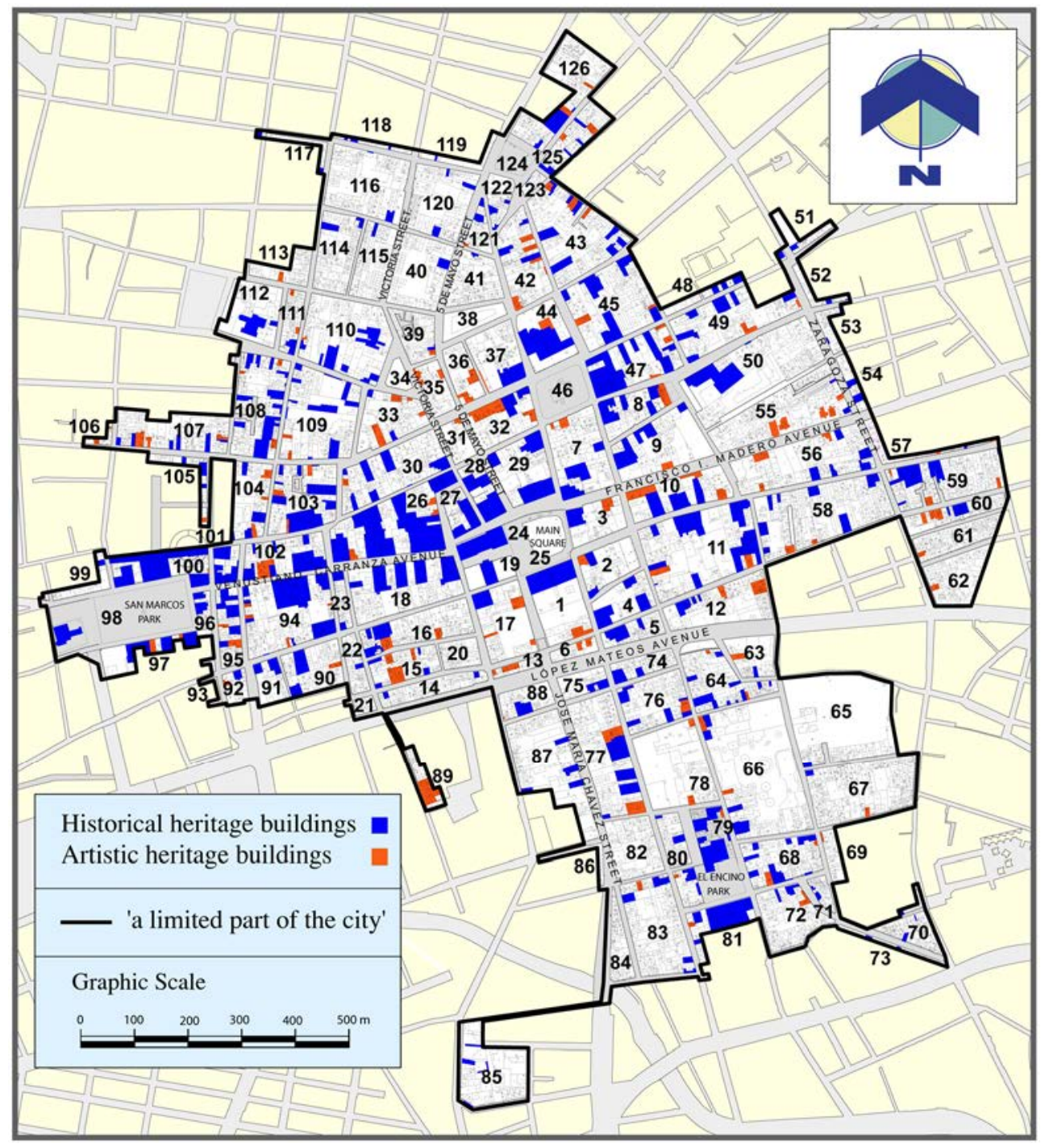

Figure 1: Architectural heritage and artistic heritage in "a limited part of the city", Aguascalientes, Ags. (Drawing made by Alejandro Acosta Collazo and Tania Pedroza, June 2019.) 
In order to classify architecture in the city centre, a border was set in the drawing as "a limited part of the city" (see Fig. 1). As the drawing made in 1855 by the geographer Isidoro Epstein was the most accurate historic plan of the city (also the oldest one), it was used to determine "a limited part of the city" of Aguascalientes now [5]. Also, in this plan we can observe two types of buildings: historic and artistic. The main difference - mentioned in the Mexican historic preservation regulations and historic data - is related with construction epochs of buildings. Thus, a unique building built during the Viceroyalty and during the 19th century is considered as part of the historical heritage list. And a unique building built during the 20th century (from the year of 1900 to the year of 2000) is considered as part of the artistic heritage list. In order to demonstrate such identifications, some areas were marked with different colours, and a new drawing was made (see Fig. 1). But it's important to mention that not all the buildings built during such periods of time are considered monuments. In order to preserve and control demolitions in the city centre, the National Institute of Anthropology and History (INAH) set a catalogue. But also throughout the development of this research, more buildings were identified - specifically those buildings with artistic values - in a cultural site worth preserving (a limited part of the city). Regardless of demolition of architecture built in the 16th and 17th centuries, some important buildings of the 18th century - like churches - still stand on the site. But, most of the buildings listed in the catalogue are civil constructions made during the 19th century. So, What are the reasons of such loss of urban heritage? The lack of regulation of land use and zoning might be the answer.

\section{ARTISTIC HERITAGE BUILDINGS AND THE 20TH CENTURY}

The official catalogue of monuments, lists a few buildings built during the 20th century. And since current Mexican laws don't support preserving buildings from such century, most of unique artistic heritage buildings are at danger of demolition. Notwithstanding old fashion regulations, researches like these could help to understand how important it is for national and local culture to document historic properties of each city block. Norman Tyler says: Documentation is also one of the preservationist's most interesting activities, for the research is like a mystery hunt - bits and pieces of information are put together like clues, leading to the answers needed to document a structure's historic significance [6]. Even though historic heritage buildings seem to be more important - in preservation terms - than artistic heritage buildings, the fact is that production of valuable architecture during the 20th century is worth preserving. Also, it's a fact that modernism changed the way we built and design architecture, but also some masterpieces of such architecture were created. So, it's time to revalue such types of buildings and set new rules for their preservation. Schools of restoration in Mexico are now producing several studies about modernism and contemporary architecture, in order to find new ways to restore them.

The lack of regulations to preserve artistic buildings is related to the lack of technics to preserve unique modern buildings. At the beginning of the 20th century constructors thought that using new materials like cement and reinforced concrete - substituting clay houses or handmade houses - was a solution for long-lasting architecture, but throughout the years we've realized that such material deterioration happened significantly faster than traditional construction materials. In that sense, restoration schools play an important role to preserve integrity and original qualities of construction materials.

In the other hand, urban mobility during the 20th century became a problem in historic centres in Mexico. In the meantime artistic heritage buildings don't have regulations to prevent their demolition, mobility require places to park cars. In fact, when Spaniards founded new cities in Mexico, houses and the whole city weren't designed to keep automotive vehicles in the city centres. Furthermore, Industrial Revolution completely 
changed the way we lived. So, there's a dilemma among parking lots, commercial shops, industry, residential buildings and sustainability of architectural heritage sites that can be analysed using statistical methods.

\section{METHODOLOGY}

Once the "limited part of the city" was defined as an area that included the most important historic heritage buildings and artistic heritage buildings in Aguascalientes City, the next step in the research was to obtain significant data, based on land areas of every property in each city block.

The drawing made by Isidoro Epstein registered the whole constructions in the small village of Aguascalientes in 1855, so nowadays we can identify - by georeferencing the polygon of the historic map with a newer one - the changes in the urban properties and land use. The main difference between the two maps is the accuracy of data in the newer drawing, however it was possible to compare valuable information. Each city block was reviewed in a Computer Aided Design (CAD) program.

Also, a cadastral drawing with boundaries of land parcels was used inside each city block to identify land uses and correct sizes of land properties. Since demolition of buildings has become a common situation inside the "limited part of the city" during the last decades a visual review was very useful to identify historical and artistic heritage buildings that are still standing.

In order to organize the information, Tables 1 and 2 were made using the following variables: city block number (previously indicated in Fig. 1), historical heritage buildings (areas and loss of architecture), area per city block, percentage of loss of buildings per city block, orchards in Epstein's drawing, gaps in city blocks in Epstein's drawing, empty areas, parking lots, commercial buildings, residential buildings, population per city block, industry land and government offices and services.

The cases of the old orchards at the end have produced empty properties where owners preferred to build parking lots or residential buildings lately. It's convenient to mention that also artistic heritage buildings replaced historical heritage buildings during the 20th century. Additionally, in the Epstein's drawing there were some gaps in the city blocks which means nothing was built in such places, as well as there were some empty areas in the new drawing, but most of the time now they use the parcels to build parking lots or as open parklands.

Since the "part of the city" selected for the research includes the historic centre of the whole city, in general, it's an important place for Aguascalientes City because part of it is considered as architectural heritage site, also because of the main administrative and government bureaus of the city are located in that place. Furthermore, new city centralities are becoming important in the city nowadays.

The UNESCO included the Royal Inland Road, also known as the Silver Route in the World Heritage List with an Outstanding Universal Value. They mention the impact of the road was enormous in terms of social tensions as well as ultimately social integration between the many people that came to be involved in the economic development. The structures in the property together reflect some aspects of this interchange of ideas and people along the southern stretch of the road [7]. As part of the Royal Inland Road, the core of Aguascalientes City centre was included, as well as San Marcos site and Carranza Av. which communicates with a road the Main Square with San Marcos site [8].

Different economic centralities in the city have produced severe problems in the historic centre lately, for example: pollution, excessive mobility, tearing down buildings and loss of heritage architecture - among others. Nowadays there are two economic poles in the city produced by the most recent centralities: Altaria Mall and NISSAN factory complex, 
Table 1: Total square meters in each city block (Part A: from 1 to 63) as a basis for a cluster analysis. It includes also useful variables to determine loss of architecture. (Source: Author's database.)

\begin{tabular}{|c|c|c|c|c|c|c|c|c|c|c|c|c|c|c|}
\hline \multirow{2}{*}{$\begin{array}{l}\text { City block } \\
\text { number }\end{array}$} & \multicolumn{2}{|c|}{ Historical heritage bulldings } & \multirow{2}{*}{$\begin{array}{c}\text { Artistic } \\
\text { heritage } \\
\text { buildings } \\
(\mathrm{m} 2)\end{array}$} & \multirow{2}{*}{$\begin{array}{l}\text { Area per } \\
\text { city block }\end{array}$} & \multirow{2}{*}{$\begin{array}{l}\text { \% Loss of } \\
\text { buildings } \\
\text { per city } \\
\text { block }\end{array}$} & \multirow{2}{*}{$\begin{array}{c}\text { Orchard in } \\
\text { Epstein's } \\
\text { drawing } \\
\text { (m2) }\end{array}$} & \multirow{2}{*}{$\begin{array}{l}\text { Gaps in city } \\
\text { blocks in } \\
\text { Epstein's } \\
\text { drawing (m2) }\end{array}$} & \multirow{2}{*}{$\begin{array}{l}\text { Empty } \\
\text { areas } \\
\text { (m2) }\end{array}$} & \multirow{2}{*}{$\begin{array}{c}\text { Parking } \\
\text { lots (m2) }\end{array}$} & & esidential & & & \\
\hline & Actual area & $\begin{array}{l}\text { Loss of } \\
\text { architecture } \\
\text { m2 }\end{array}$ & & & & & & & & buildings (m2) & $\begin{array}{l}\text { Dildings } \\
(\mathrm{m} 2)\end{array}$ & per city block & land (m2) & intices and $(\mathrm{m} 2)$ \\
\hline No_Manz & $m_{2}, P$ Arq & m2,P_Arq_ perd & m2_p_Art & $m_{2}$ Manz & $\begin{array}{c}\text { \%perd_ } \\
\text { Manz }\end{array}$ & $\begin{array}{l}\text { m2_Huet- } \\
\text { Epstein - }\end{array}$ & $\begin{array}{l}\text { m2_ Huecos_ } \\
\text { Epstein }\end{array}$ & $\begin{array}{c}\mathrm{m} 2 \\
\text { Baddios }\end{array}$ & $\begin{array}{c}\mathrm{m}^{\mathrm{m} 2} \text { - } \\
\text { Estacionam } \\
\text { lento }\end{array}$ & $\begin{array}{l}\text { m2, Fine- } \\
\text { Comerc }\end{array}$ & m2_Finc, $\mathrm{Hab}$ & $\begin{array}{c}\text { hebilantes, Me } \\
n z\end{array}$ & $\begin{array}{l}\text { m2 } \\
\text { industria }\end{array}$ & $\begin{array}{c}\text { M2_Senvidios } \\
\text { Officinas }\end{array}$ \\
\hline 1 & 6672.30 & 5008.24 & 685.49 & 12366.03 & 0.40 & 0.00 & 0.00 & 0.00 & 2460.03 & 1276.50 & 895.02 & 20.00 & 0.00 & 6790.78 \\
\hline 2 & 637.14 & 6367.76 & 280.42 & 7285.32 & 0.87 & 0.00 & 0.00 & 0.00 & \begin{tabular}{|l|}
1503.07 \\
\end{tabular} & 297.29 & 637.34 & 34.00 & 0.00 & 1575.72 \\
\hline 3 & 845.45 & 5765.26 & 301.24 & 6911.95 & 0.83 & 0.00 & 0.00 & 0.00 & 599.62 & 1648.56 & 0.00 & 0.00 & 0.00 & 1644.80 \\
\hline 4 & 2023.80 & 5174.75 & 0.00 & 7198.55 & 0.72 & 0.00 & 0.00 & 0.00 & 3017.28 & 498.42 & 505.00 & 17.00 & 0.00 & 493.67 \\
\hline 5 & 1102.96 & 3291.29 & 0.00 & 439427 & 0.75 & 0.00 & 0.00 & 0.00 & 0.00 & 3291.29 & 1102.98 & 41.00 & 0.00 & 0.00 \\
\hline 6 & 0.00 & 1884.49 & 358.54 & 224303 & 0.84 & 0.00 & 0.00 & 0.00 & 0.00 & 1010.50 & 1043.65 & 9.00 & 0.00 & 0.00 \\
\hline 7 & 827.36 & 13503.15 & 334.76 & 14665.27 & 0.92 & 0.00 & 0.00 & 0.00 & 0.00 & 14070.19 & 595.08 & 3.00 & 0.00 & 0.00 \\
\hline 8 & 2145.79 & 5760.56 & 356.09 & 8262.44 & 0.70 & 0.00 & 0.00 & 0.00 & 0.00 & 1743.05 & 2499.14 & 13.00 & 0.00 & 0.00 \\
\hline 9 & 1815.26 & 4046.22 & 154.89 & 15698.02 & 0.26 & 9681.65 & 0.00 & 1401.64 & 2878.07 & 5536.51 & 1112.23 & 21.00 & 0.00 & 0.00 \\
\hline 10 & 2391.03 & 3168.56 & 1374.75 & 11309.53 & 0.28 & 4375.19 & 0.00 & 428.69 & 406.27 & 5803.84 & 1405.45 & 27.00 & 0.00 & 2287.64 \\
\hline 11 & 5437.36 & 20399.53 & 694.45 & 28422.18 & 0.72 & 0.00 & 0.00 & 1890.84 & 3217.64 & 5207.31 & 11532.71 & 114.00 & 0.00 & 770.50 \\
\hline 12 & 704.14 & 6610.88 & 946.14 & 13362.07 & 0.49 & 5100.91 & 0.00 & 1917.13 & 1248.44 & 27770.18 & 6716.44 & 74.00 & 0.00 & 709.88 \\
\hline 13 & 0.00 & 1479.61 & 199.67 & 1679.28 & 0.88 & 0.00 & 0.00 & 0.00 & 0.00 & 1057.53 & 621.75 & 0.00 & 0.00 & 0.00 \\
\hline 14 & 234.38 & 5815.68 & 0.00 & 6050.06 & 0.96 & 0.00 & 0.00 & 0.00 & 0.00 & 3073.90 & 2910.12 & 94.00 & 0.00 & 66.04 \\
\hline 15 & 496.91 & 5209.73 & 1231.78 & 6938.44 & 0.75 & 0.00 & 0.00 & 0.00 & 595.54 & 118.90 & 6224.00 & 83.00 & 0.00 & 0.00 \\
\hline 16 & 1465.30 & 9851.49 & 258.13 & 11574.92 & 0.85 & 0.00 & 0.00 & 0.00 & 426.64 & 5906.91 & 5241.37 & 76.00 & 0.00 & 0.00 \\
\hline 17 & 926.63 & 10222.30 & 1134.83 & 12283.73 & 0.83 & 0.00 & 0.00 & 206.93 & 0.00 & 3638.25 & 1660.20 & 29.00 & 0.00 & 2223.29 \\
\hline 18 & 4811.68 & 13568.85 & 0.00 & 18380.53 & 0.74 & 0.00 & 0.00 & 0.00 & 0.00 & 11545.76 & 2552.12 & 66.00 & 0.00 & 0.00 \\
\hline 19 & 1030.74 & 2796.56 & 0.00 & 3827.30 & 0.73 & 0.00 & 0.00 & 0.00 & 0.00 & 688.35 & 678.62 & 0.00 & 0.00 & 1429.59 \\
\hline 20 & 0.00 & 3677.70 & 0.00 & 3677.70 & 1.00 & 0.00 & 0.00 & 138.27 & 0.00 & 590.54 & 2298.52 & 76.00 & 0.00 & 788.64 \\
\hline 21 & 0.00 & 1153.11 & 0.00 & 1153.11 & 1.00 & 0.00 & 0.00 & 0.00 & 0.00 & 890.29 & 262.82 & 22.00 & 0.00 & 0.00 \\
\hline 22 & 143.80 & 3279.34 & 0.00 & 3423.14 & 0.96 & 0.00 & 0.00 & 27.95 & 0.00 & 243.48 & 3179.66 & 51.00 & 0.00 & 0.00 \\
\hline 23 & 495.23 & 1952.07 & 0.00 & 2447.30 & 0.80 & 0.00 & 0.00 & 0.00 & 0.00 & 328.88 & 2118.42 & 17.00 & 0.00 & 0.00 \\
\hline 24 & 2772.31 & 0.00 & 0.00 & 4673.05 & 0.00 & 0.00 & 0.00 & 0.00 & 0.00 & 0.00 & 0.00 & 0.00 & 0.00 & 0.00 \\
\hline 25 & 0.00 & 0.00 & 0.00 & 10615.01 & 0.00 & 0.00 & 0.00 & 0.00 & 0.00 & 0.00 & 0.00 & 0.00 & 0.00 & 0.00 \\
\hline 26 & 11010.75 & 10161.24 & 395.25 & 21567.24 & 0.47 & 0.00 & 0.00 & 96.55 & 281.46 & 4074.24 & 11073.41 & 94.00 & 0.00 & 0.00 \\
\hline 27 & 1560.22 & 2468.22 & 0.00 & 4028.44 & 0.61 & 0.00 & 0.00 & 0.00 & 0.00 & 2262.97 & 1309.92 & 22.00 & 0.00 & 455.55 \\
\hline 28 & 2785.81 & 2134.50 & 0.00 & 4920.31 & 0.43 & 0.00 & 0.00 & 0.00 & 0.00 & 2617.84 & 112.70 & 7.00 & 0.00 & 2189.77 \\
\hline 29 & 3463.86 & 7487.80 & 0.00 & 10951.66 & 0.68 & 0.00 & 0.00 & 0.00 & 0.00 & 7993.73 & 0.00 & 0.00 & 0.00 & 1846.22 \\
\hline 30 & 2529.53 & 13193.08 & 138.79 & 15861.40 & 0.83 & 0.00 & 0.00 & 0.00 & 312.83 & 9115.52 & 6433.05 & 130.00 & 0.00 & 0.00 \\
\hline 31 & 0.00 & 3255.63 & 115.77 & 3371.40 & 0.97 & 0.00 & 0.00 & 0.00 & 0.00 & 3255.63 & 115.77 & 11.00 & 0.00 & 0.00 \\
\hline 32 & 1098.68 & 6357.05 & 1608.95 & 906468 & 0.70 & 0.00 & 0.00 & 0.00 & 0.00 & 6279.82 & 361.20 & 4.00 & 0.00 & 1628.39 \\
\hline 33 & 317.57 & 9203.52 & 551.60 & 10072.69 & 0.91 & 0.00 & 0.00 & 0.00 & 879.90 & 7758.56 & 1434.23 & 62.00 & 0.00 & 0.00 \\
\hline 34 & 0.00 & 1849.80 & 0.00 & 1849.80 & 1.00 & 0.00 & 0.00 & 0.00 & 0.00 & 0.00 & 0.00 & 0.00 & 0.00 & 0.00 \\
\hline 35 & 0.00 & 3920.51 & 804.32 & 4724.83 & 0.83 & 0.00 & 0.00 & 0.00 & 0.00 & 4223.77 & 254.66 & 31.00 & 0.00 & 246.40 \\
\hline 36 & 0.00 & 4634.57 & 511.24 & 5844.97 & 0.79 & 0.00 & 0.00 & 0.00 & 1609.29 & 3025.28 & 511.24 & 2.00 & 0.00 & 0.00 \\
\hline 37 & 1345.49 & 7680.51 & 401.20 & 9427.29 & 0.81 & 0.00 & 0.00 & 0.00 & 0.00 & 9427.29 & 0.00 & 3.00 & 0.00 & 0.00 \\
\hline 38 & 0.00 & 4445.74 & 0.00 & 4445.74 & 1.00 & 0.00 & 0.00 & 0.00 & 0.00 & 0.00 & 0.00 & 0.00 & 0.00 & 0.00 \\
\hline 39 & 114.49 & 5960.50 & 68.74 & 6143.73 & 0.97 & 0.00 & 0.00 & 0.00 & 0.00 & 6143.73 & 0.00 & 11.00 & 0.00 & 0.00 \\
\hline 40 & 367.03 & 7774.28 & 0.00 & 12564.17 & 0.62 & 0.00 & 0.00 & 0.00 & 4422.86 & 8141.31 & 0.00 & 15.00 & 0.00 & 0.00 \\
\hline 41 & 65.20 & 7725.13 & 0.00 & 7790.33 & 0. & 0.0 & 0.00 & 0.00 & 0.00 & 7210.21 & 155.81 & 14.00 & 0.00 & 424.31 \\
\hline 42 & 845.07 & 9575.24 & 1189.74 & 11610.05 & 0.82 & 0.0 & 0.00 & 0.00 & 2213.96 & 7088.82 & 1919.42 & 43.00 & 0.00 & 387.85 \\
\hline 43 & 2513.35 & 16781.22 & 909.97 & 20204.54 & 0. & 0.00 & 0.0 & 232.12 & 6278.51 & 8322.33 & 5371.58 & 125.00 & 0.00 & 0.00 \\
\hline 44 & 3970.07 & 394.82 & 394.01 & 9750.93 & 0.04 & 3913.46 & 0.00 & 0.00 & 0.00 & 413.86 & 0.00 & 1.00 & 0.00 & 225.05 \\
\hline 45 & 3381.26 & 15365.66 & 535.39 & 1928231 & 0.80 & 0.00 & 0.00 & 0.00 & 3233.10 & 9746.53 & 4686.25 & 100.00 & 0.00 & 389.51 \\
\hline 46 & 0.00 & 6528.60 & 0.00 & 6528.60 & 1.00 & 0.00 & 0.00 & 0.00 & 0.00 & 0.00 & 0.00 & 0.00 & 0.00 & 0.00 \\
\hline 47 & 5718.97 & 6960.42 & 947.16 & 13626.55 & 0.51 & 0.00 & 0.00 & 89.90 & 0.00 & 7933.15 & 4517.90 & 90.00 & 0.00 & 0.00 \\
\hline 48 & 629.75 & 1997.75 & 0.00 & 2627.50 & 0.76 & 0.0 & 0.00 & 0.00 & 0.00 & 1385.35 & 1242.15 & 80.00 & 0.00 & 0.00 \\
\hline 49 & 2028.80 & 13652.10 & 716.26 & 16397.16 & 0.83 & 0.00 & 0.00 & 0.00 & 1035.88 & 9688.42 & 3072.86 & 85.00 & 0.00 & 0.00 \\
\hline 50 & 3064.92 & 11321.64 & 0.00 & 24776.15 & 0.46 & 10389.59 & 0.00 & 0.00 & 0.00 & 3825.96 & 4738.01 & 68.00 & 0.00 & 0.00 \\
\hline 51 & 141.84 & & 0.0 & 2761.12 & 0.95 & 0.00 & 0.00 & 0.00 & 0.0 & 567.14 & 797.38 & 24.00 & 0.00 & 0.00 \\
\hline 52 & 130.87 & 1675.27 & 0.00 & 1806.14 & 0.93 & 0.00 & 0.00 & 0.00 & 110.13 & 1298.23 & 397.78 & 48.00 & 0.00 & 0.00 \\
\hline 53 & 53.41 & 2075.26 & 0.0 & 2128.67 & 0.9 & $0 . c$ & 0.00 & 0.00 & 0.00 & 574.48 & 1219.26 & 45.00 & 0.00 & 334.93 \\
\hline 54 & 499.85 & 1771.61 & 0.00 & 2271.46 & 0.78 & 0.00 & 0.00 & 0.00 & 0.00 & 798.61 & 1472.85 & 36.00 & 0.00 & 0.00 \\
\hline 55 & 220.51 & 1469.53 & 122.46 & 1989621 & 0.07 & 18083.71 & 0.00 & 0.00 & 0.00 & 1109.91 & 455.95 & 119.00 & 0.00 & 0.00 \\
\hline 56 & 1654.55 & 13795.79 & 1918.60 & 19778.59 & 0.7 & 0.00 & 0.0 & 0.00 & 2409.65 & 12488.63 & 4880.31 & 48.00 & 0.00 & 0.00 \\
\hline 57 & 47.09 & 2868.51 & 173.32 & 3088.92 & 0.93 & 0.00 & 0.00 & 0.00 & 99.37 & 1276.69 & 1712.86 & 64.00 & 0.00 & 0.00 \\
\hline 58 & 2650.46 & 20318.79 & 327.13 & 25404.65 & 0.80 & 0.00 & 0.00 & 0.00 & 2108.27 & 727.77 & 20086.10 & 263.00 & 0.00 & 1196.81 \\
\hline 59 & 1406.16 & 62 & 281.33 & 16398.77 & 0.38 & 8716.94 & 0.00 & 0.00 & 0.00 & 1162.67 & 6088.27 & 140.00 & 0.00 & 0.00 \\
\hline 60 & 0.00 & 1567.12 & 0.00 & 5934.55 & 0.26 & 4367.43 & 0.00 & 0.00 & 0.00 & 387.10 & 1217.99 & 119.00 & 0.00 & 0.00 \\
\hline 61 & 0.0 & 2054 & 192. & 7096 & 0 & & $0 . c$ & 0.00 & 0.0 & 802.00 & 1449.49 & 103.00 & 0.00 & 0.00 \\
\hline 62 & 0.00 & 4680.12 & 204.48 & 8702.61 & 0.54 & 3818.01 & 0.00 & 0.00 & 336.79 & 2566.34 & 489.01 & 24.00 & 0.00 & 0.00 \\
\hline 63 & 292.81 & 3123.90 & 256.71 & 5912.27 & 0.53 & 0.00 & 0.00 & 2238.85 & 252.93 & 1492.32 & 1928.17 & 35.00 & 0.00 & 0.00 \\
\hline
\end{tabular}

producing also urban changes inside the historic centrality [9]. The main problem of the historic centrality is that originally it wasn't designed for motorized mobility. Also, there's a lack of regulations to protect heritage buildings from demolition. Thus, in order to take advantage of the historic site, commercial shops remain frequently in historic buildings.

Commercial activities and services located in a historic site always require mobility. But in this case, parking lots are useful for commercial shops and the mobility system. So, parking lots located close to the commercial places increase mobility.

The amount of cars circulating in the historic centre of Aguascalientes make more difficult to find a spot to park in the streets when someone drives, so, in general, most of the people 
Table 2: Total square meters in each city block (Part B: from 64 to 126) as a basis for a cluster analysis. It includes also useful variables to determine loss of architecture. (Source: Author's database.)

\begin{tabular}{|c|c|c|c|c|c|c|c|c|c|c|c|c|c|c|}
\hline \multirow{2}{*}{$\begin{array}{l}\text { City block } \\
\text { number }\end{array}$} & \multicolumn{2}{|c|}{ Historical heritage bulldings } & \multirow{2}{*}{ 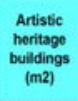 } & \multirow{2}{*}{$\begin{array}{l}\text { Area por } \\
\text { city block }\end{array}$} & \multirow{2}{*}{$\begin{array}{l}\text { \% Loss of } \\
\text { buildings } \\
\text { per city } \\
\text { block }\end{array}$} & \multirow{2}{*}{$\begin{array}{l}\text { Orchard in } \\
\text { Epstoin's } \\
\text { drawing } \\
\text { (m2) }\end{array}$} & \multirow{2}{*}{$\begin{array}{l}\text { Gaps in city } \\
\text { blocks in } \\
\text { Epstein's } \\
\text { drawing ( } \mathrm{m} 2 \text { ) }\end{array}$} & \multirow{2}{*}{$\begin{array}{l}\text { Empty } \\
\text { areas } \\
\text { (m2) }\end{array}$} & & & & & & \\
\hline & Actual area & $\begin{array}{c}\text { Loss of } \\
\text { architecture } \\
\text { m2 }\end{array}$ & & & & & & & $\begin{array}{l}\text { Pats }(\mathrm{m} 2) \\
\text { lots }\end{array}$ & buildi & $\begin{array}{l}\text { buildings } \\
\text { (m2) }\end{array}$ & $\begin{array}{l}\text { ation } \\
\text { block }\end{array}$ & $\begin{array}{l}\text { Industry } \\
\text { land }(\mathrm{m} 2)\end{array}$ & $\begin{array}{l}\text { officos and } \\
\text { services (m2) }\end{array}$ \\
\hline No_Manz & $m 2$ P_Arq & m2_P_AvQ_perd & me_p_Ant & m2_Manz & $\begin{array}{l}\text { \%.Perd_M } \\
\text { anz }\end{array}$ & $\begin{array}{l}\text { m2_Huer- } \\
\text { Epstein }\end{array}$ & $\begin{array}{l}\text { m2 Huecos_ } \\
\text { Epstein }\end{array}$ & Baldios & $\begin{array}{c}\mathrm{m}_{2} \\
\text { Estacionam } \\
\text { iento }\end{array}$ & $\begin{array}{l}\mathrm{m}_{2} \text {. Finc_- } \\
\text { Comerc }\end{array}$ & $\mathrm{m}_{2}$. Finc_Hab & $\begin{array}{l}\text { habitantes } \\
\text { Mare }\end{array}$ & $\begin{array}{l}\text { m2 } \\
\text { Industria }\end{array}$ & $\begin{array}{c}\text { m2_Servicios } \\
\text { OOficnas }\end{array}$ \\
\hline 64 & 1476.83 & 9333.09 & 418.92 & 12798.60 & 0.73 & 0.00 & 0.00 & 0.00 & 1569.76 & 1147.17 & 8228.95 & 98.00 & 0.00 & 586.72 \\
\hline 65 & 95.51 & 20721.71 & 0.00 & 29614.28 & 0.70 & 8797.06 & 0.00 & 0.00 & 16065.32 & 785.08 & 5931.88 & 120.00 & 6831.50 & 0.00 \\
\hline 66 & 1886.33 & 14526.75 & 111.58 & 37386.09 & 0.39 & 20861.43 & 0.00 & 0.00 & 479.88 & 2098.20 & 7137.01 & 56.00 & 4329.87 & 0.00 \\
\hline 67 & 155.63 & 15217.77 & 1064.36 & 19930.88 & 0.76 & 0.00 & 0.00 & 0.00 & 1523.12 & 1358.98 & 15078.78 & 206.00 & 1969.81 & 0.00 \\
\hline 68 & 2046.78 & 7688.67 & 512.20 & 10758.10 & 0.71 & 0.00 & 0.00 & 510.45 & 0.00 & 1474.73 & 6411.12 & 71.00 & 0.00 & 0.00 \\
\hline 69 & 89.07 & 3353.92 & 61.67 & 3504.66 & 0.96 & 0.00 & 0.00 & 0.00 & 455.43 & 88.66 & 2960.57 & 52.00 & 0.00 & 0.00 \\
\hline 70 & 159.98 & 4488.69 & 0.00 & 4648.67 & 0.97 & 0.00 & 0.00 & 0.00 & 0.00 & 38.37 & 4610.30 & 80.00 & 0.00 & 0.00 \\
\hline 71 & 206.38 & 1665.52 & 0.00 & 1871.90 & 0.89 & 0.00 & 0.00 & 0.00 & 0.00 & 42.52 & 1829.38 & 44.00 & 0.00 & 0.00 \\
\hline 72 & 438.08 & 7707.12 & 470.46 & 10657.66 & 0.72 & 0.00 & 0.00 & 0.00 & 0.00 & 1033.21 & 7582.45 & 71.00 & 2041.61 & 0.00 \\
\hline 73 & 172.39 & 1082.11 & 0.00 & 1254.50 & 0.86 & 0.00 & 0.00 & 0.00 & 0.00 & 306.42 & 948.08 & 22.00 & 0.00 & 0.00 \\
\hline 74 & 790.31 & 3468.52 & 0.00 & 4258.83 & 0.81 & 0.00 & 0.00 & 224.16 & 0.00 & 1685.91 & 2348.76 & 36.00 & 0.00 & 0.00 \\
\hline 75 & 639.53 & 3102.63 & 0.00 & 3742.16 & 0.83 & 0.00 & 0.00 & 0.00 & 0.00 & 2778.23 & 963.93 & 11.00 & 0.00 & 0.00 \\
\hline 76 & 1461.81 & 6865.60 & 385.09 & 10936.06 & 0.63 & 0.00 & 0.00 & 0.00 & 2223.56 & 484.96 & 8227.54 & 71.00 & 0.00 & 0.00 \\
\hline 77 & 2891.42 & 14713.10 & 1530.23 & 19134.75 & 0.77 & 0.00 & 0.00 & 0.00 & 525.61 & 11657.91 & 6951.23 & 103.00 & 0.00 & 0.00 \\
\hline 78 & 0.00 & 18239.14 & 393.40 & 18632.54 & 0.98 & 0.00 & 0.00 & 0.00 & 0.00 & 0.00 & 3226.39 & 34.00 & 15406.15 & 0.00 \\
\hline 79 & 4237.84 & 4802.00 & 151.12 & 14394.96 & 0.33 & 0.00 & 0.00 & 0.00 & 0.00 & 347.41 & 5362.27 & 57.00 & 0.00 & 0.00 \\
\hline 80 & 351.19 & 3850.26 & 0.00 & 4201.45 & 0.92 & 0.00 & 0.00 & 0.00 & 0.00 & 82.55 & 4118.90 & 36.00 & 0.00 & 0.00 \\
\hline 81 & 3886.90 & 2226.62 & 111.75 & 6225.27 & 0.36 & 0.00 & 0.00 & 0.00 & 328.81 & 1828.89 & 3322.29 & 82.00 & 70.28 & 0.00 \\
\hline 82 & 327.32 & 5853.16 & 1306.68 & 9623.70 & 0.61 & 0.00 & 0.00 & 0.00 & 2136.54 & 2050.57 & 5436.59 & 24.00 & 0.00 & 0.00 \\
\hline 83 & 1232.23 & 12008.99 & 165.85 & 17803.85 & 0.67 & 0.00 & 3542.80 & 0.00 & 853.98 & 672.25 & 11911.26 & 124.00 & 0.00 & 0.00 \\
\hline 84 & 0.00 & 4547.84 & 0.00 & 4547.84 & 1.00 & 0.00 & 0.00 & 149.45 & 0.00 & 2064.51 & 2333.88 & 66.00 & 0.00 & 0.00 \\
\hline 85 & 316.41 & 14276.56 & 0.00 & 14592.97 & 0.98 & 0.00 & 0.00 & 0.00 & 0.00 & 2335.54 & 8383.57 & 25.00 & 0.00 & 1029.37 \\
\hline 86 & 0.00 & 1263.15 & 0.00 & 1263.15 & 1.00 & 0.00 & 0.00 & 0.00 & 0.00 & 793.65 & 469.50 & 24.00 & 0.00 & 0.00 \\
\hline 87 & 1152.24 & 8643.00 & 0.00 & 20193.66 & 0.43 & 0.00 & 3355.50 & 917.88 & 7042.92 & 4976.44 & 3900.92 & 102.00 & 0.00 & 0.00 \\
\hline 88 & 683.24 & 4160.27 & 0.00 & 4843.51 & 0.86 & 0.00 & 0.00 & 0.00 & 0.00 & 3371.08 & 1472.43 & 29.00 & 0.00 & 0.00 \\
\hline 89 & 0.00 & 1815.63 & 1222.02 & 3037.65 & 0.60 & 0.00 & 0.00 & 0.00 & 0.00 & 0.00 & 3037.65 & 48.00 & 0.00 & 0.00 \\
\hline 90 & 1342.59 & 7354.91 & 145.71 & 8843.21 & 0.83 & 0.00 & 0.00 & 0.00 & 301.70 & 1926.15 & 5587.28 & 56.00 & 0.00 & 935.76 \\
\hline 91 & 743.45 & 3307.32 & 0.00 & 4050.77 & 0.82 & 0.00 & 0.00 & 0.00 & 0.00 & 136.61 & 3689.65 & 54.00 & 0.00 & 0.00 \\
\hline 92 & 239.78 & 2123.82 & 108.64 & 2472.24 & 0.86 & 0.00 & 0.00 & 0.00 & 0.00 & 361.85 & 2110.39 & 20.00 & 0.00 & 0.00 \\
\hline 93 & 0.00 & 543.22 & 0.00 & 543.22 & 1.00 & 0.00 & 0.00 & 0.00 & 227.60 & 199.10 & 344.12 & 10.00 & 0.00 & 0.00 \\
\hline 94 & 5647.55 & 8087.97 & 325.79 & 20697.47 & 0.39 & 0.00 & 5106.49 & 0.00 & 1529.60 & 5327.26 & 8644.86 & 71.00 & 0.00 & 0.00 \\
\hline 95 & 1744.46 & 3816.10 & 415.98 & 5976.54 & 0.64 & 0.00 & 0.00 & 0.00 & 219.66 & 2214.24 & 3542.64 & 64.00 & 0.00 & 0.00 \\
\hline 96 & 666.52 & 992.64 & 0.00 & 1659.16 & 0.60 & 0.00 & 0.00 & 0.00 & 0.00 & 264.43 & 1394.73 & 25.00 & 0.00 & 0.00 \\
\hline 97 & 2555.23 & 3744.55 & 362.17 & 6661.95 & 0.56 & 0.00 & 0.00 & 0.00 & 0.00 & 155.03 & 2204.52 & 24.00 & 0.00 & 1063.09 \\
\hline 98 & 1114.65 & 0.00 & 0.00 & 22934.84 & 0.00 & 0.00 & 0.00 & 0.00 & 0.00 & 0.00 & 0.00 & 0.00 & 0.00 & 0.00 \\
\hline 99 & 159.87 & 2918.00 & 0.00 & 3077.87 & 0.95 & 0.00 & 0.00 & 0.00 & 106.56 & 2685.52 & 285.79 & 16.00 & 0.00 & 0.00 \\
\hline 100 & 6329.31 & 3863.78 & 0.00 & 10193.09 & 0.38 & 0.00 & 0.00 & 0.00 & 291.61 & 7520.15 & 1739.33 & 45.00 & 0.00 & 0.00 \\
\hline 101 & 1952.46 & 1089.59 & 0.00 & 3042.05 & 0.36 & 0.00 & 0.00 & 0.00 & 0.00 & 1739.23 & 1302.82 & 4.00 & 0.00 & 0.00 \\
\hline 102 & 3084.20 & 4901.00 & 816.55 & 10030.60 & 0.49 & 0.00 & 1228.85 & 0.00 & 0.00 & 4786.73 & 3331.26 & 68.00 & 0.00 & 0.00 \\
\hline 103 & 4070.96 & 6639.51 & 507.41 & 14414.19 & 0.46 & 0.00 & 3196.31 & 0.00 & 0.00 & 1705.96 & 11106.59 & 123.00 & 0.00 & 0.00 \\
\hline 104 & 1026.28 & 3941.21 & 599.83 & 9009.72 & 0.44 & 0.00 & 344240 & 0.00 & 0.00 & 730.30 & 4837.02 & 88.00 & 0.00 & 0.00 \\
\hline 105 & 547.80 & 2493.77 & 110.18 & 3151.75 & 0.79 & 0.00 & 0.00 & 0.00 & 0.00 & 440.29 & 2711.46 & 62.00 & 0.00 & 0.00 \\
\hline 106 & 0.00 & 820.14 & 118.40 & 938.54 & 0.87 & 0.00 & 0.00 & 0.00 & 77.75 & 171.45 & 689.34 & 8.00 & 0.00 & 0.00 \\
\hline 107 & 1076.87 & 8248.24 & 890.25 & 13639.61 & 0.60 & 0.00 & 3424.25 & 89.35 & 0.00 & 331.46 & 9270.54 & 93.00 & 0.00 & 0.00 \\
\hline 108 & 3678.53 & 7830.57 & 343.49 & 1642684 & 0.48 & 0.00 & 4574.25 & 0.00 & 150.32 & 255462 & 13189.90 & 140.00 & 532.47 & 0.00 \\
\hline 109 & 2134.03 & 14022.67 & 234.84 & 20857.46 & 0.67 & 0.00 & 4465.92 & 0.00 & 7525.32 & 8748.30 & 3924.62 & 84.00 & 0.00 & 0.00 \\
\hline 110 & 2713.06 & 18342.54 & 46.81 & 21102.41 & 0.87 & 0.00 & 0.00 & 240.85 & 2829.90 & 16557.30 & 1111.26 & 65.00 & 363.07 & 0.00 \\
\hline 111 & 406.64 & 3428.78 & 181.71 & 3835.42 & 0.89 & 0.00 & 0.00 & 0.00 & 0.00 & 689.72 & 3145.70 & 85.00 & 0.00 & 0.00 \\
\hline 112 & 1345.22 & 7625.18 & 0.00 & 9995.40 & 0.76 & 0.00 & 0.00 & 0.00 & 0.00 & 401.44 & 933.46 & 41.00 & 0.00 & 0.00 \\
\hline 113 & 0.00 & 4301.07 & 65.55 & 4386.62 & 0.98 & 0.00 & 0.00 & 0.00 & 0.00 & 3274.23 & 1092.39 & 89.00 & 0.00 & 0.00 \\
\hline 114 & 58638 & 6254.92 & 0.00 & 10332.08 & 0.61 & 2608.36 & 882.42 & 642.48 & 0.00 & 6962.34 & 2149.13 & 113.00 & 578.41 & 0.00 \\
\hline 115 & 17230 & 4648.67 & 0.00 & 8304.09 & 0.56 & 3483.12 & 0.00 & 0.00 & 0.00 & 6042.90 & 2261.19 & 72.00 & 0.00 & 0.00 \\
\hline 116 & 309.34 & 6616.26 & 0.00 & 17207.27 & 0.38 & 5599.16 & 4682.51 & 0.00 & 2634.93 & 5974.10 & 8598.24 & 176.00 & 0.00 & 0.00 \\
\hline 117 & 217.39 & 1495.43 & 0.00 & 1712.82 & 0.87 & 0.00 & 0.00 & 0.00 & 0.00 & 434.12 & 1278.70 & 44.00 & 0.00 & 0.00 \\
\hline 118 & 190.20 & 999.20 & 0.00 & 1189.40 & 0.84 & 0.00 & 0.00 & 0.00 & 0.00 & 407.04 & 574.46 & 42.00 & 0.00 & 0.00 \\
\hline 119 & 52.62 & 2093.35 & 0.00 & 2145.97 & 0.98 & 0.00 & 0.00 & 0.00 & 0.00 & 1466.70 & 52.62 & 42.00 & 0.00 & 626.65 \\
\hline 120 & 487.32 & 6419.88 & 330.40 & 13950.45 & 0.46 & 5218.19 & 1825.06 & 155.71 & 3287.34 & 9433.44 & 487.24 & 62.00 & 0.00 & 586.72 \\
\hline 121 & 345.13 & 2546.23 & 46.62 & 2937.98 & 0.87 & 0.00 & 0.00 & 0.00 & 0.00 & 2937.98 & 0.00 & 8.00 & 0.00 & 0.00 \\
\hline 122 & 353.16 & 3408.04 & 0.00 & 3761.20 & 0.91 & 0.00 & 0.00 & 177.91 & 0.00 & 3427.73 & 155.56 & 10.00 & 0.00 & 0.00 \\
\hline 123 & 0.00 & 1058.93 & 0.00 & 1058.93 & 1.00 & 0.00 & 0.00 & 0.00 & 484.77 & 333.43 & 240.73 & 13.00 & 0.00 & 0.00 \\
\hline 124 & 0.00 & 0.00 & 0.00 & 4006.60 & 0.00 & 0.00 & 0.00 & 0.00 & 0.00 & 0.00 & 0.00 & 1.00 & 0.00 & 0.00 \\
\hline 125 & 588.83 & 5429.87 & 509.21 & 6527.91 & 0.83 & 0.00 & 0.00 & 0.00 & 436.30 & 185.20 & 5906.91 & 73.00 & 0.00 & 0.00 \\
\hline 126 & 1369.59 & 12292.86 & 379.46 & 14041.91 & 0.88 & 0.00 & 0.00 & 0.00 & 1997.55 & 7861.98 & 3019.98 & 94.00 & 0.00 & 0.00 \\
\hline TOTaLs & 162837.10 & 754433.73 & 35520.55 & 1192410.27 & 0.63 & 119863.75 & 39728.78 & 11777.11 & 97243.37 & 378314.21 & 388398.28 & 6730.00 & 32123.17 & 32983.65 \\
\hline
\end{tabular}

who visit and drive to historic places have to look for a parking lot. Some people preferred to drive a car to go to the historic centre instead of taking a bus [10].

Most of the historical heritage buildings indicated in Fig. 1 are registered in a catalogue list of the INAH, but there's still land speculation inside the "limited part of the city", so some owners prefer to let their building become obsolete by doing nothing about their preservation, and the historic constructions slowly deteriorate. Scarcity becomes a factor to 
appreciate the land in value over time. So, when the building deteriorates too much and becomes useless and risky for inhabitants, authorities allow demolition.

After analysing the "limited part of the city" and significant variables (see Tables 1 and 2) a multivariate statistical method was applied, particularly a cluster analysis was used to identify homogenous groups of categories. Tables 1 and 2 were made using square meters of each variable inside the "limited part of the city". In this case, it wasn't necessary to use a sample, because the whole data inside the area were analysed; thus, it was considered as a census. The program SPSS $®$ Statistics was very useful in applying a cluster analysis. Also, more information about land use was applied, but the tables weren't included in this paper.

\section{RESULTS}

The multivariate statistical method was effective to understand deterioration of the historic centre. After running a hierarchical cluster analysis, the city blocks (Tables 1 and 2) were linked to similar situations and a dendrogram of cluster analysis using Ward linkage was produced (see Fig. 2). We can notice - in the tree-based representation of the observations that four clusters were produced. The city blocks of each cluster have something to do with each other, but this will be demonstrated in the conclusions. In the results, first of all it's convenient to mention what's happening with the parking lots and mobility.

It can be observed in the Plot box, Parking lots of Fig. 2, that the city block number 65 has the most room for parking lots with $16,065.32 \mathrm{~m}^{2}$. This is the case of a factory that locates in the old quarter: El Encino and keeps plenty of parking spots for its employees and the trucks they use to transport merchandise routinely. Of course this is part of the mobility system that requires a big factory. The contrast of this situation is that the historic quarter wasn't designed originally for this type of land use. In fact originally it used to be a residential place and also there were some orchards.

Also, inside the city block number 109 , the area of parking lots is $7,525.32 \mathrm{~m}^{2}$. This city block is close to commercial shops that have become a profitable centrality lately in the whole city. But, in this case mobility and such centrality have become a dilemma because of high loss of historic buildings; in brief, $14,022.67 \mathrm{~m}^{2}$ that means $67 \%$ of the architecture that used to be in such city block in the year of 1855 .

Another atypical case of parking lots growth places inside the city block number 87 in which the loss of architecture was $8,643.00 \mathrm{~m}^{2}$ (43\% of original buildings). This city block is located in a commercial pole of the city. In fact, it's difficult to find historic heritage buildings in this city block nowadays.

In accordance with the Ward method (see Fig. 2), the last atypical case shown in the Plot box - parking lots was the city block number 43 . This city block keeps only $2,513.35 \mathrm{~m}^{2}$ of historical heritage buildings and the loss of architecture was $83 \%$, which means $20,204.54$ $\mathrm{m}^{2}$. In this case parking lots include an extent of $6,278.51 \mathrm{~m}^{2}$, but a lot of changes in buildings have occurred since 1855 .

Paradigmatic incidents and radical changes of architecture can be demonstrated in city block number 38 as well as in city block number 46 . In the first case there used to stand the oldest market in town - named Mercado Juárez, but in the year of 1981 a fired burned out part of it and the administrators of the City Hall decided to tear it down. In order to replace the old building they built a new market with a parking lot, including low-level designs.

In the case of city block number 46 , there used to be an old building with commercial shops, but in the years of 1985 and 1986 the proprietors demolished the historic building and built a new commercial place. Also, this building included low-level three story parking lots. These buildings were required those days by the increasing mobility in the city - especially in the core of the city - to give room to automobiles parking spaces. 


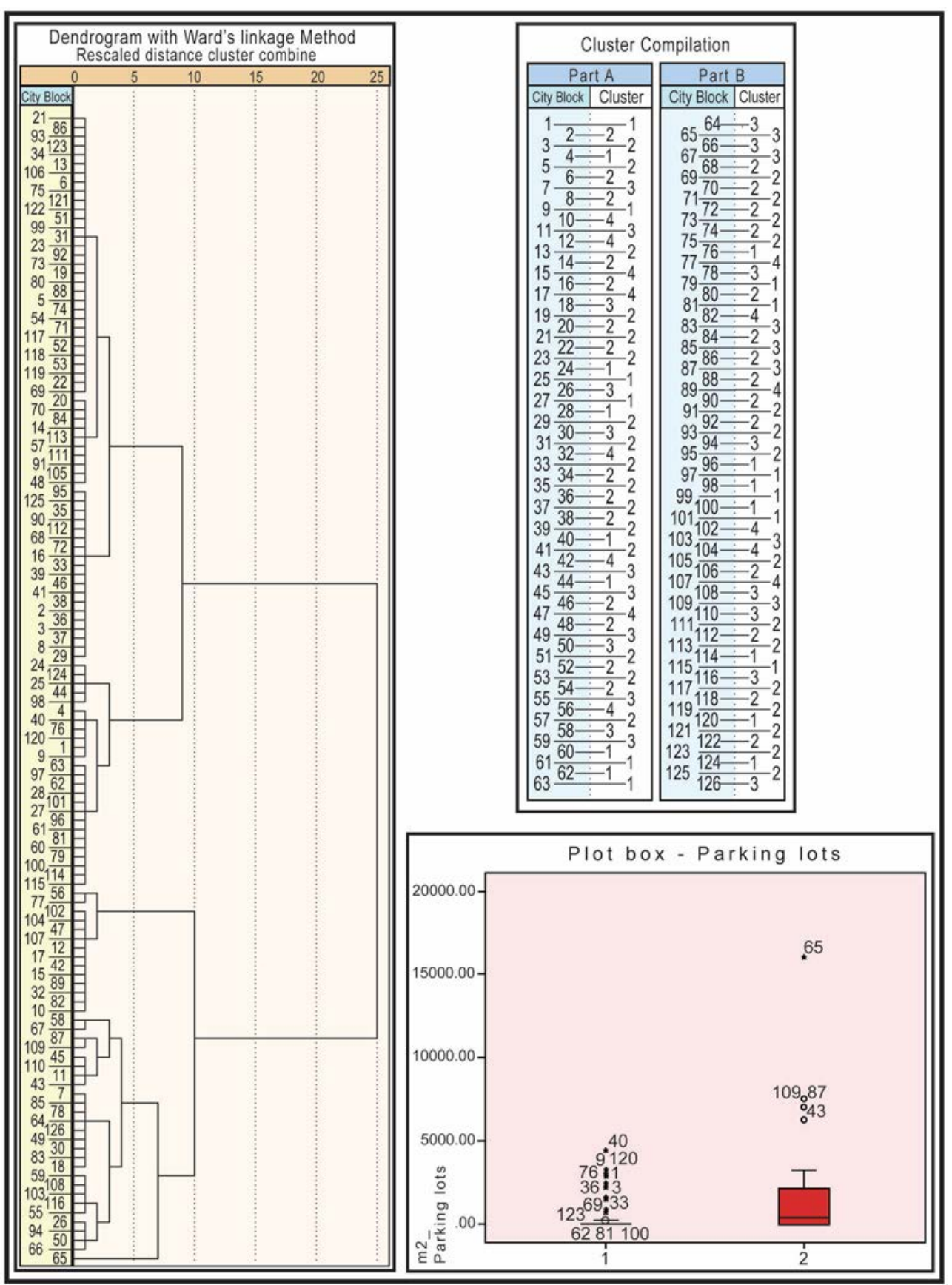

Figure 2: Dendrogram with Ward's Linkage Method. Rescaled distance cluster combine, cluster compilation and Plot box of parking lots. (Calculations made by Alejandro Acosta, July 2019.) 
The Ward method in Fig. 2 demonstrates in the Plot box - parking lots, that city blocks number: 40, 9 and 120 express similarities. Also the three cases linked each other in cluster 1 (see Fig. 3). The city block number 40 is part of a commercial centrality of the city and it only preserves $367.03 \mathrm{~m}^{2}$ of historic heritage buildings. So, there's a loss of $62 \%$ of the original buildings in such city block. The parking area is $4,422.86 \mathrm{~m}^{2}$. Similarly, city block number 120 is strongly linked to the adjacent commercial shops. In this case the loss of historical heritage buildings is $46 \%$. Finally, street block number 9 keeps $1,815.26 \mathrm{~m}^{2}$ of historical heritage buildings and the loss of it is $26 \%$. But the area of the parking lots is $2,878.07 \mathrm{~m}^{2}$.

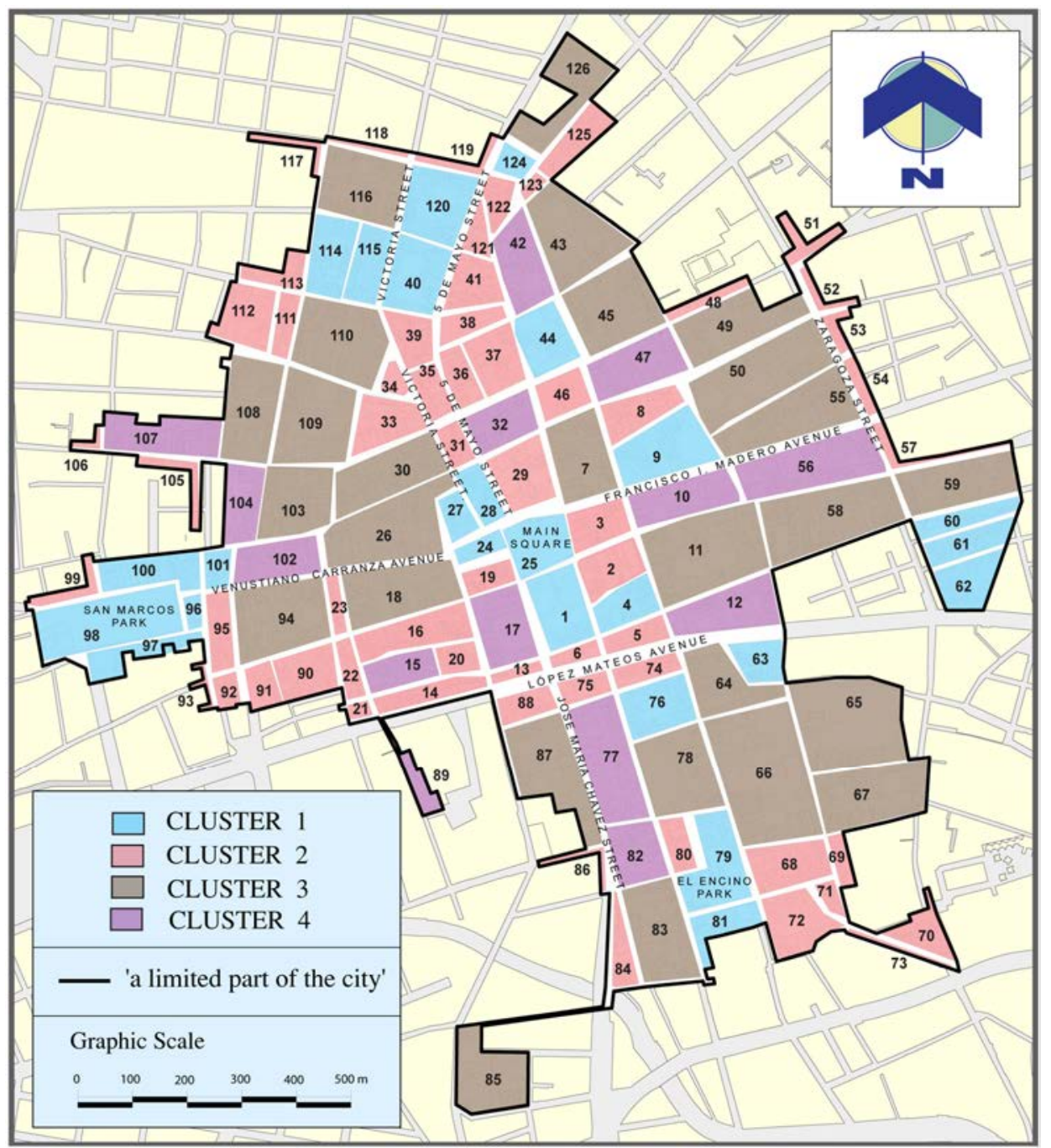

Figure 3: Rescaled distance cluster combine in "a limited part of the city" drawing, based on cluster compilation. (Calculations made by Alejandro Acosta Collazo, July 2019. Drawing made by Tania Pedroza and Alejandro Acosta Colunga, July 2019.) 


\section{CONCLUSIONS}

In order to represent the cluster compilation a drawing was made using the "limited part of the city" of Aguascalientes (see Fig. 3). The plot box of the parking lots (see Fig. 2) demonstrated the city blocks number: $65,109,87$ and 43 were atypical. The main cause of it was the size of the parking lots.

In the case of cluster 1 (see Fig. 3) the relationship between historic heritage buildings and open spaces is high, for example inside the city block 25 the Main Square is located. Analogously inside the city block 98 the San Marcos Park can be found and inside the city block 79 El Encino Park is situated. Also inside the city block number 1 the Garden of the Palaces can be found.

In cluster number 2, most of the city blocks keep commercial shops. But, it's remarkable because it highlights loss of architecture and a pattern related to urban mobility on principal streets in the "limited part of the city", like López Mateos Av., Zaragoza Street, 5 de Mayo Street and Victoria Street. All these streets show economic prosperity, nevertheless, there appears to be a dilemma: the government and society have to decide now between the importance of preserving historic heritage buildings or making economic profits. As a consequence, more parking lots were built to support the increasing mobility in the city. Also, loss of historic architecture was more common in the oldest street blocks of the city; consequently, in the historic site there's a lack of architecture built during the 16th and 17th centuries.

Cluster 3 shows similarities on the amount and sizes of historic heritage buildings. Also, it includes most of the city blocks $(7,18,26,30,94,103,108$ and 7) of the architectural heritage site in the city centre, as part of the Silver Route in the UNESCO World Heritage List.

At the end of the analysis, cluster 4 shows more isolated city blocks, and some of such city blocks keep artistic heritage buildings, for example inside city blocks 10 and 56 there are good examples of modern buildings built during the 20th century. Nowadays, it's hard to find a perfectly preserved 19th century house inside the "limited part of the city" in Aguascalientes. But, there have been noticeable efforts from the city governors to preserve the architecture constructed by Refugio Reyes, whom was the most important architect at the beginning of the 20th century, for example San Antonio church, which is a unique artistic heritage building.

The loss of architecture significance draws attention and shows that sustainability of architectural heritage is at risk. So, city management planning should become a priority in Mexico to improve historic sites. Also, an adequate zoning is necessary to develop cultural sustainability in Mexico.

Maybe is time to think about some other possibilities to enhance preservation of historic buildings in Mexico, for example by promoting tourism and values of architecture, instead of so many commercial shops, in order to contribute to progress of social and cultural balances; thus, become a sustainable city.

In fact, tourism is one of the main economic sectors in Mexico; thus, it embodies $8.7 \%$ of Gross Domestic Product (GDP) [11] and it continues to grow every year. Also, the Mexican architectural sites could promote tourism in order to increase incomes for restoration of unique buildings.

Finally, the use of a multivariate statistical method, specially a cluster exploration, and the use of history of urbanism and architecture, contributed in this paper to determine critical alterations of historical heritage buildings and artistic heritage buildings in the "limited part of the city". 


\section{ACKNOWLEDGEMENTS}

I thank my research assistants: Jéssica Alejandra Rodríguez Torres, Tania Judith Pedroza Díaz and Alejandro Acosta Colunga. I also thank the students: Getsemani Díaz Barriga, Fernando Jesús May Vázquez, Ricardo Daniel Alfaro Ake, Norma Elena Alemán López, Sandra Nallely González Robles, Leopoldo Madrigal Muñoz, Mauricio Rivera Garza and María Fernanda Pérez Sifuentes. Appreciation is extended to my colleague and researcher: Marco Alejandro Sifuentes Solís.

\section{REFERENCES}

[1] Powell, P.W., The Chichimeca War (1150-1600), Fondo de Cultura Económica: Mexico City, pp. 213-231, 1996.

[2] Acosta Collazo, A., Mexican fortresses built in the 16th century. Morphology of maritime heritage and historic arsenals preserved. WIT Transactions on Ecology and The Environment, vol. 193, WIT Press: Southampton and Boston, p. 632, 2015.

[3] Acosta Collazo, A., Historic preservation in Aguascalientes, Mexico: Urban regeneration, new corporate centralities and sustainability. WIT Transactions on The Built Environment, vol. 153, WIT Press: Southampton and Boston, p. 126, 2015.

[4] Acosta Collazo, A., St. Nicholas of Tolentino monastery and church in Actopan, Hidalgo: Preservation, surveying and reuse of a heritage building in Mexico. WIT Transactions on the Built Environment, vol. 118, WIT Press: Southampton and Boston, p. 172, 2011.

[5] Acosta Collazo, A., Mobility and collateral effects on city heritage in Aguascalientes, Mexico. WIT Transactions on The Built Environment, vol. 179, WIT Press: Southampton and Boston, p. 58, 2018.

[6] Tyler, N., Historic Preservation. An Introduction to its History, Principles, and Practice, W.W. Norton, p. 93, 2000.

[7] Camino Real de Tierra Adentro, World Heritage List, UNESCO. whc.unesco.org/en/list/1351/. Accessed on: 6 Jul. 2019.

[8] Camino Real de Tierra Adentro, World Heritage List, UNESCO, map location. whc.unesco.org/en/list/1351/multiple=1\&unique_number=1691. Accessed on: $6 \mathrm{Jul}$. 2019.

[9] Acosta Collazo, A., Historic preservation in Aguascalientes, Mexico: Urban regeneration, new corporate centralities and sustainability. WIT Transactions on The Built Environment, vol. 153, WIT Press: Southampton and Boston, p. 133, 2015.

[10] Acosta Collazo, A., Urban mobility and qualitative research in historic places. WIT Transactions on The Built Environment, vol. 182, WIT Press: Southampton and Boston, p. 278, 2019.

[11] Bureau of Tourism (Sectur), Institute of Touristic Competitiveness and Knowledge Management Department, Researchers ICTUR, 2019. 